Supporting information for

\title{
An efficient synthesis of quaterrylenedicarboximide NIR dyes
}

\author{
Yuri Avlasevich and Klaus Müllen*
}

Max Planck Institute for Polymer Research,

Ackermannweg 10, D-55128 Mainz, Germany

muellen@mpip-mainz.mpg.de

Table of Contents

General Section

S2

Procedures

S3 - S5

References

S5

NMR Spectra

S6 -S12 


\section{General Section}

The solvents and chemicals used were of commercial grade. Compounds 2a-c were synthesised as described elsewhere. ${ }^{1,2}$ Column chromatography was performed on silica gel (Silicagel 60A, 0.06-0.2 mm, Acros). Melting points were performed on a Büchi MP B-545 melting point apparatus and are not corrected. Thermal gravimetric analyses were performed using Mettler TGA851e apparatus. ${ }^{1} \mathrm{H}$ and ${ }^{13} \mathrm{C}$ NMR spectra were recorded on Bruker DPX250, Bruker AMX300, Bruker DRX500 and Bruker WS700 spectrometers. Infrared spectra were obtained on a Nicolet FT IR320. FD mass spectra were recorded with a VG-instruments ZAB 2-SE-FPD instrument. MALDI-TOF mass spectra were recorded on a Bruker Reflex II-TOF spectrometer. UV/Vis spectra were recorded on a Perkin-Elmer Lambda 900 spectrophotometer. Fluorescence spectra were recorded on a Spex Fluorolog 3 spectrometer. Fluorescence quantum yield of compound $\mathbf{4 b}$ was determined by the relative method using Rhodamine 800 as a reference $\left(\phi_{\mathrm{F}}=39 \%\right.$ in dichloroethane $\left.{ }^{3}\right)$. 


\section{Procedures}

$N$-(2,6-diisopropylphenyl)-9-(perylen-3-yl)-perylene-3,4-dicarboximide (7a). $N$-(2,6diisopropylphenyl)-9-bromoperylene-3,4-dicarboximide (0.56 g, $1 \mathrm{mmol})$ and compound 6 $(0.57 \mathrm{~g}, 1.5 \mathrm{mmol})$ were dissolved in the mixture of toluene $(50 \mathrm{~mL})$ and ethanol $(2 \mathrm{~mL})$ in 100 $\mathrm{mL}$ Schlenk flask and flushed with argon. After stirring at $70{ }^{\circ} \mathrm{C}$ for 20 min $2 \mathrm{M}$ aqueous $\mathrm{K}_{2} \mathrm{CO}_{3}(2 \mathrm{~mL})$ and $\mathrm{Pd}\left(\mathrm{PPh}_{3}\right)_{4}(40 \mathrm{mg}, 0.04 \mathrm{mmol})$ were added to the solution. The reaction mixture was stirred at $95{ }^{\circ} \mathrm{C}$ for $20 \mathrm{~h}$ under argon. After cooling the resulting mixture was washed with water and extracted with toluene. The combined organic extracts were evaporated in vacuo and purified by column chromatography on silica gel using dichloromethane as an eluent. Yield $527 \mathrm{mg}$ (72\%). M. p. $>350{ }^{\circ} \mathrm{C} ; \mathbf{R}_{\mathbf{f}}=0.63$ (dichloromethane); ${ }^{1} \mathbf{H}-\mathbf{N M R}(500 \mathrm{MHz}$, $\left.\mathbf{C}_{2} \mathbf{D}_{2} \mathbf{C l}_{4}, \mathbf{1 0 0}{ }^{\circ} \mathbf{C}\right): \delta=8.63\left(\mathrm{~d},{ }^{3} \mathrm{~J}=8.0 \mathrm{~Hz}, 1 \mathrm{H}\right), 8.59\left(\mathrm{~d},{ }^{3} \mathrm{~J}=7.6 \mathrm{~Hz}, 1 \mathrm{H}\right), 8.53\left(\mathrm{t},{ }^{3} \mathrm{~J}=8.1 \mathrm{~Hz}, 1 \mathrm{H}\right)$, $8.47\left(\mathrm{t},{ }^{3} \mathrm{~J}=8.1 \mathrm{~Hz}, 2 \mathrm{H}\right), 8.44\left(\mathrm{~d},{ }^{3} J=7.1 \mathrm{~Hz}, 1 \mathrm{H}\right), 8.13-8.34(\mathrm{~m}, 3 \mathrm{H}), 7.89\left(\mathrm{~d},{ }^{3} \mathrm{~J}=8.0 \mathrm{~Hz}, 1 \mathrm{H}\right)$, 7.78-7.83 (m, 1H), $7.71\left(\mathrm{dd},{ }^{3} J=7.7 \mathrm{~Hz}, 2 \mathrm{H}\right), 7.63\left(\mathrm{dd},{ }^{3} J=8.0 \mathrm{~Hz}, 2 \mathrm{H}\right), 7.48-7.56(\mathrm{~m}, 3 \mathrm{H}), 7.39$ (t, $\left.{ }^{3} \mathrm{~J}=8.1 \mathrm{~Hz}, 2 \mathrm{H}\right), 7.32$ (s, 1H), 7.28 (d, $\left.{ }^{3} \mathrm{~J}=7.8 \mathrm{~Hz}, 2 \mathrm{H}\right), 2.75$ (sep, $\left.{ }^{3} \mathrm{~J}=7.3 \mathrm{~Hz}, 2 \mathrm{H}\right), 1.16$ (dd, $\left.{ }^{3} \mathrm{~J}=6.4 \mathrm{~Hz}, 12 \mathrm{H}\right) \mathrm{ppm} ;{ }^{13} \mathbf{C}-\mathbf{N M R}\left(\mathbf{1 7 5} \mathbf{M H z}, \mathbf{C}_{2} \mathbf{D}_{\mathbf{2}} \mathbf{C l}_{\mathbf{4}}, \mathbf{1 0 0}{ }^{\circ} \mathbf{C}\right): 163.6,146.8,137.4,131.6$, 131.4, 131.3, 130.4, 128.9, 128.3, 127.1, 127.0, 123.6, 121.3, 29.4, 29.0, 23.73, 23.71 ppm; IR (KBr): $v=3425,2924,1701,1659,1592,1580,1357,1243,1109 \mathrm{~cm}^{-1}$; UV-Vis (chlorobenzene): $\lambda_{\max }$ (rel. int.): 522 (1), 496 (1), $449(0.76), 428(0.55) \mathrm{nm}$; fluorescence (chlorobenzene): $\lambda_{\max }: 683 \mathrm{~nm}$; MS (MALDI-TOF): $\mathrm{m} / \mathrm{z}$ (rel. int.) 731 (100\%), $\mathrm{M}^{+}$; Elemental analysis: Calcd for $\mathrm{C}_{54} \mathrm{H}_{37} \mathrm{NO}_{2}$ : C, 88.62; H, 5.10; N, 1.91. Found: C, 88.54; H, $5.04 ; \mathrm{N}, 1.95 \%$.

$N$-(2,6-diisopropylphenyl)quaterrylene-3,4-dicarboximide (4a). Compound 7a (0.1 g, $0.137 \mathrm{mmol})$ was dissolved in dry dichloromethane $(40 \mathrm{~mL})$ in $100 \mathrm{~mL}$ Shlenk flask. After stirring for 20 min under argon a solution of anhydrous iron (III) chloride (1 g) in dry nitromethane $(4 \mathrm{~mL})$ was added through a syringe. The solution was stirred at room temperature for $72 \mathrm{~h}$ under slow flow of argon. Dry methanol $(100 \mathrm{~mL})$ was added to the solution. The resulting precipitate was filtered, rinsed with methanol and purified by the precipitation from chlorobenzene into methanol. Yield $80 \mathrm{mg}(80 \%)$. M. p. $>350{ }^{\circ} \mathrm{C}$; IR (KBr): $v=3448,2958,2923,1697,1655,1570,1545,1353,1243,809 \mathrm{~cm}^{-1}$; MS (MALDI-TOF): m/z (rel. int.) 729 (100\%), M+; \%); UV-Vis (chlorobenzene): $\lambda_{\max }$ (rel. int.): 740 (1), 670 (0.49), $612(0.3) \mathrm{nm}$. 
$N$-(2,6-diisopropylphenyl)-1,6-bis(4-(1,1,3,3-tetramethylbutyl)phenoxy)-9-(9(10)-

bromoperylen-3-yl)-perylene-3,4-dicarboximide (7c). $N$-(2,6-diisopropylphenyl)-1,6-bis(4(1,1,3,3-tetramethylbutyl)phenoxy)-9-(4,4,5,5-tetramethyl-1,3,2-dioxaborolan-2-yl)-perylene3,4-dicarboximide (0.51 g, $0.5 \mathrm{mmol})$ and 3,9(10)-dibromoperylene (1.43 g, $3.5 \mathrm{mmol})$ were dissolved in the mixture of toluene $(40 \mathrm{~mL})$ and ethanol $(2 \mathrm{~mL})$ in $100 \mathrm{~mL}$ Schlenk flask and flushed with argon. After stirring at $70{ }^{\circ} \mathrm{C}$ for 20 min $2 \mathrm{M}$ aqueous $\mathrm{K}_{2} \mathrm{CO}_{3}(0.5 \mathrm{~mL})$ and $\mathrm{Pd}\left(\mathrm{PPh}_{3}\right)_{4}(30 \mathrm{mg}, 0.03 \mathrm{mmol})$ were added to the solution. The reaction mixture was stirred at $70{ }^{\circ} \mathrm{C}$ for $6 \mathrm{~h}$ under argon. After cooling the resulting mixture was washed with water and extracted with toluene. The combined organic extracts were evaporated in vacuo and purified by column chromatography on silica gel using toluene as an eluent. Yield $420 \mathrm{mg}$ (69\%). M. p. $310{ }^{\circ} \mathrm{C} ; \mathbf{R}_{\mathbf{f}}=0.72$ (toluene); ${ }^{\mathbf{H}} \mathbf{H}-\mathbf{N M R}\left(250 \mathbf{M H z}, \mathbf{C D}_{\mathbf{2}} \mathbf{C l}_{\mathbf{2}}, 25{ }^{\circ} \mathbf{C}\right): \delta=9.48\left(\mathrm{~d},{ }^{3} \mathrm{~J}=8.0 \mathrm{~Hz}, 1 \mathrm{H}\right)$, $9.35\left(\mathrm{~d},{ }^{3} \mathrm{~J}=7.6 \mathrm{~Hz}, 1 \mathrm{H}\right), 8.20-8.40(\mathrm{~m}, 2 \mathrm{H}), 8.26(\mathrm{~s}, 1 \mathrm{H}), 8.24(\mathrm{~s}, 1 \mathrm{H}), 8.09\left(\mathrm{t},{ }^{3} \mathrm{~J}=8.1 \mathrm{~Hz}, 2 \mathrm{H}\right)$, $7.80\left(\mathrm{~d},{ }^{3} \mathrm{~J}=8.1 \mathrm{~Hz}, 1 \mathrm{H}\right), 7.64\left(\mathrm{dd},{ }^{3} J=8.0 \mathrm{~Hz}, 3 \mathrm{H}\right), 7.51-7.54(\mathrm{~m}, 2 \mathrm{H}), 7.44\left(\mathrm{~d},{ }^{3} \mathrm{~J}=8.7 \mathrm{~Hz}, 4 \mathrm{H}\right)$, $7.32\left(\mathrm{~d},{ }^{3} \mathrm{~J}=7.5 \mathrm{~Hz}, 4 \mathrm{H}\right), 7.06-7.35$ (m, 6H), 2.74 (sep, $\left.{ }^{3} \mathrm{~J}=6.8 \mathrm{~Hz}, 2 \mathrm{H}\right), 1.75$ (s, 2H), 1.74 (s, 2H), 1.39 (s, 6H), $1.38(\mathrm{~s}, 6 \mathrm{H}), 1.12\left(\mathrm{~d},{ }^{3} \mathrm{~J}=6.6 \mathrm{~Hz}, 12 \mathrm{H}\right), 0.73(\mathrm{~s}, 9 \mathrm{H}), 0.72(\mathrm{~s}, 9 \mathrm{H}) \mathrm{ppm} ;{ }^{13} \mathrm{C}-$ NMR (62,5 MHz, $\left.\mathbf{C D}_{2} \mathbf{C l}_{2}, 25{ }^{\circ} \mathbf{C}\right): 163.9,154.4,153.9,147.2,147.0,146.7,131.4,130.2$, $129.5,128.6,128.4,127.3,124.6,119.0,118.8,57.6,38.8,32.1,31.9,29.6,24.3$ ppm; IR (KBr): $v=3439,2958,2923,1702,1664,1588,1502,1321,1272,1209,806 \mathrm{~cm}^{-1}$; UV-Vis (toluene): $\lambda_{\max }$ (rel. int.): 524 (1), 491 (0.7), 447 (0.5), 419 (0.47) nm; MS (FD): m/z (rel. int.) 1220.6 (100\%), $\mathrm{M}^{+}$; Elemental analysis: Calcd for $\mathrm{C}_{82} \mathrm{H}_{76} \mathrm{BrNO}_{4}$ : C, 80.77; H, 6.28; N, 1.15 . Found: C, 80.71; H, 6.19; N, $1.12 \%$.

\section{$N$-(2,6-diisopropylphenyl)-1,6-bis(4-(1,1,3,3-tetramethylbutyl)phenoxy)-13-}

bromoquaterrylene-3,4-dicarboximide (4c). $\quad N$-(2,6-diisopropylphenyl)-1,6-bis(4-(1,1,3,3tetramethylbutyl)phenoxy)-9-(9(10)-bromoperylen-3-yl)-perylene-3,4-dicarboximide $\quad(0.2 \mathrm{~g}$, $0.16 \mathrm{mmol})$ was dissolved in dry dichloromethane $(10 \mathrm{~mL})$ in $25 \mathrm{~mL}$ Shlenk flask. After stirring for 20 min under argon a solution of anhydrous iron (III) chloride in dry nitromethane $(2 \mathrm{~mL})$ was added through a syringe. The solution was stirred at room temperature for $3 \mathrm{~h}$ under the slow flow of argon. Dry methanol $(50 \mathrm{~mL})$ was added to the solution. The resulting precipitate was filtered, rinsed with water and dried. The precipitate was purified by the column chromatography on silica gel using toluene as eluent. Yield $120 \mathrm{mg}(60 \%)$. M. p. $>350{ }^{\circ} \mathrm{C} ; \mathbf{R}_{\mathbf{f}}$

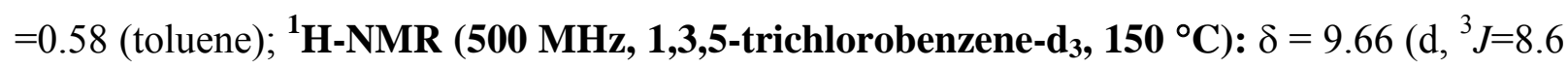
$\mathrm{Hz}, 2 \mathrm{H}), 8.58$ (s, 2H), 8.45 (d, $\left.{ }^{3} \mathrm{~J}=8.7 \mathrm{~Hz}, 2 \mathrm{H}\right), 8.30-8.39$ (m, 6H), $8.21\left(\mathrm{~d},{ }^{3} \mathrm{~J}=8.2 \mathrm{~Hz}, 1 \mathrm{H}\right), 8.07$ $\left(\mathrm{d},{ }^{3} J=7.96 \mathrm{~Hz}, 1 \mathrm{H}\right), 7.64\left(\mathrm{t},{ }^{3} J=7.7 \mathrm{~Hz}, 1 \mathrm{H}\right), 7.53\left(\mathrm{~d},{ }^{3} J=8.3 \mathrm{~Hz}, 4 \mathrm{H}\right), 7.42\left(\mathrm{t},{ }^{3} J=7.7 \mathrm{~Hz}, 1 \mathrm{H}\right)$, $7.32\left(\mathrm{~d},{ }^{3} \mathrm{~J}=7.8 \mathrm{~Hz}, 2 \mathrm{H}\right), 7.30\left(\mathrm{~d},{ }^{3} \mathrm{~J}=9.0 \mathrm{~Hz}, 4 \mathrm{H}\right), 3.00\left(\mathrm{sep},{ }^{3} \mathrm{~J}=6.8 \mathrm{~Hz}, 2 \mathrm{H}\right), 1.88$ (s, 4H), 1.51 
(s, 12H), 1.29 (dd, $\left.{ }^{3} \mathrm{~J}=6.8 \mathrm{~Hz}, 12 \mathrm{H}\right), 0.93$ (s, 9H), 0.92 (s, 9H) ppm; ${ }^{1} \mathbf{H}-{ }^{1} \mathbf{H}$ COSY Experiment (500 MHz, 1,3,5-trichlorobenzene-d $\left.\mathbf{d}_{3}, \mathbf{1 5 0}^{\circ} \mathbf{C}\right)$ : Coupling of $\delta=(9.66,8.45),(8.36,8.32)$, (8.32, 7.64), (8.21, 7.64), (7.53, 7.30), (7.42, 7.32); IR (KBr): $v=3438,2958,2923,1702$, 1664, 1588, 1502, 1321,1272, 1209, $808 \mathrm{~cm}^{-1}$; UV-Vis (dichloromethane): $\lambda_{\max }(\varepsilon): 732$ (145000), 668 (58000), 359 (14200) nm; MS (FD): m/z (rel. int.) 1218.8 (100\%), $\mathrm{M}^{+}, 609.9$ (40\%), M/2; Elemental analysis: Calcd for $\mathrm{C}_{82} \mathrm{H}_{74} \mathrm{BrNO}_{4}$ : C, 80.90; H, 6.13; N, 1.15. Found: C, 80.83; H, 6.10; N, $1.18 \%$.

\section{References:}

1. Pschirer, N. G.; Kohl, C.; Nolde, F.; Qu, J.; Müllen, K. Angew. Chem. 2006, 118, 1429-1432; Angew. Chem Int. Ed. 2006, 45, 1401-1404.

2. Weil, T., Reuther, E., Beer, C., Müllen, K. Chem. Eur. J., 2004, 10, 1398-1414.

3. Imasaka, T., Tsukamoto, A., Ishibashi, N. Anal. Chem. 1989, 61, 2285 - 2288. 

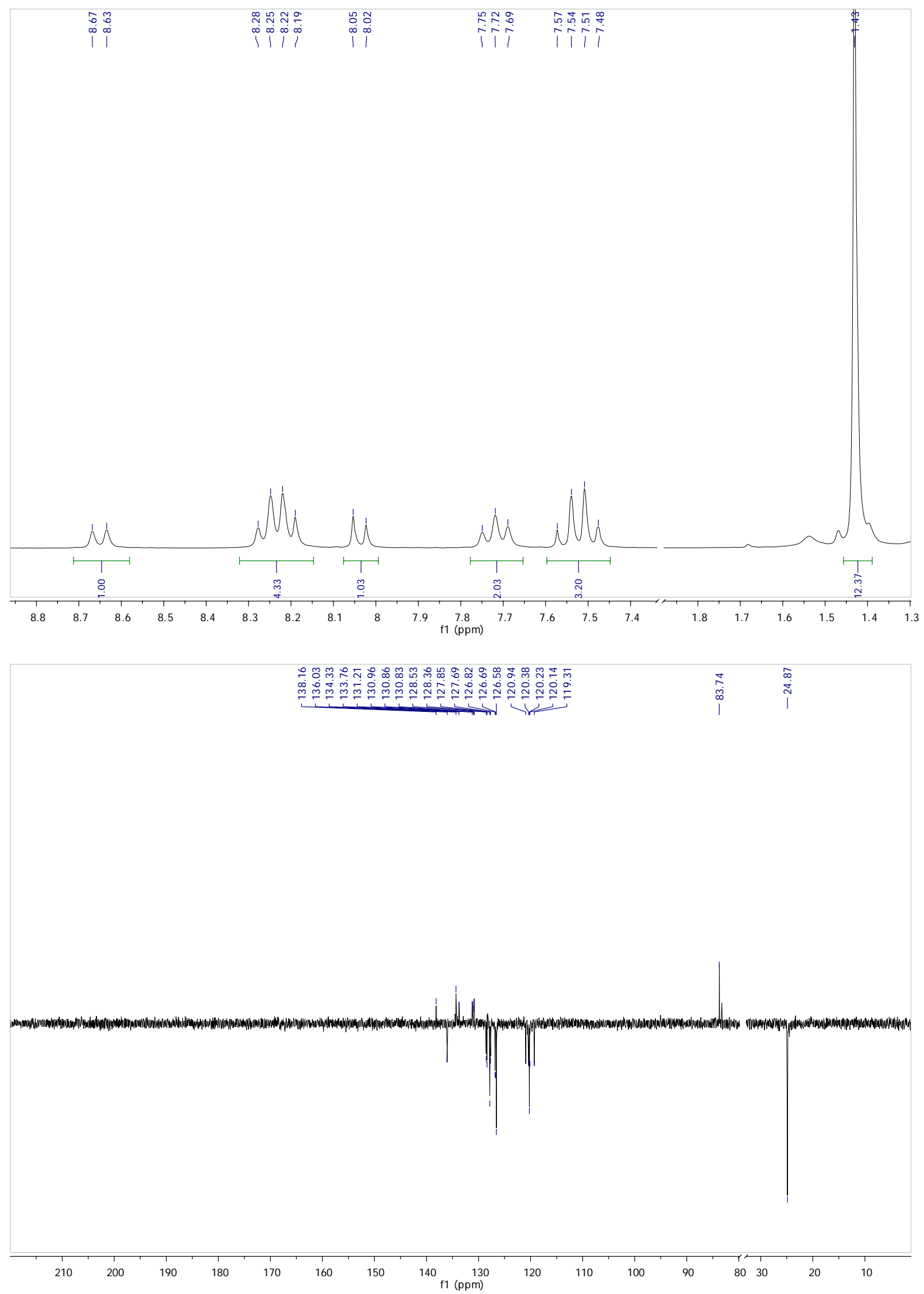

Figure S1. ${ }^{1} \mathrm{H}-\mathrm{NMR}\left(250 \mathrm{MHz}, \mathrm{CD}_{2} \mathrm{Cl}_{2}\right)$ and ${ }^{13} \mathrm{C}-\mathrm{NMR}\left(75 \mathrm{MHz}, \mathrm{C}_{2} \mathrm{D}_{2} \mathrm{Cl}_{4}\right)$ spectra of compound 6. 

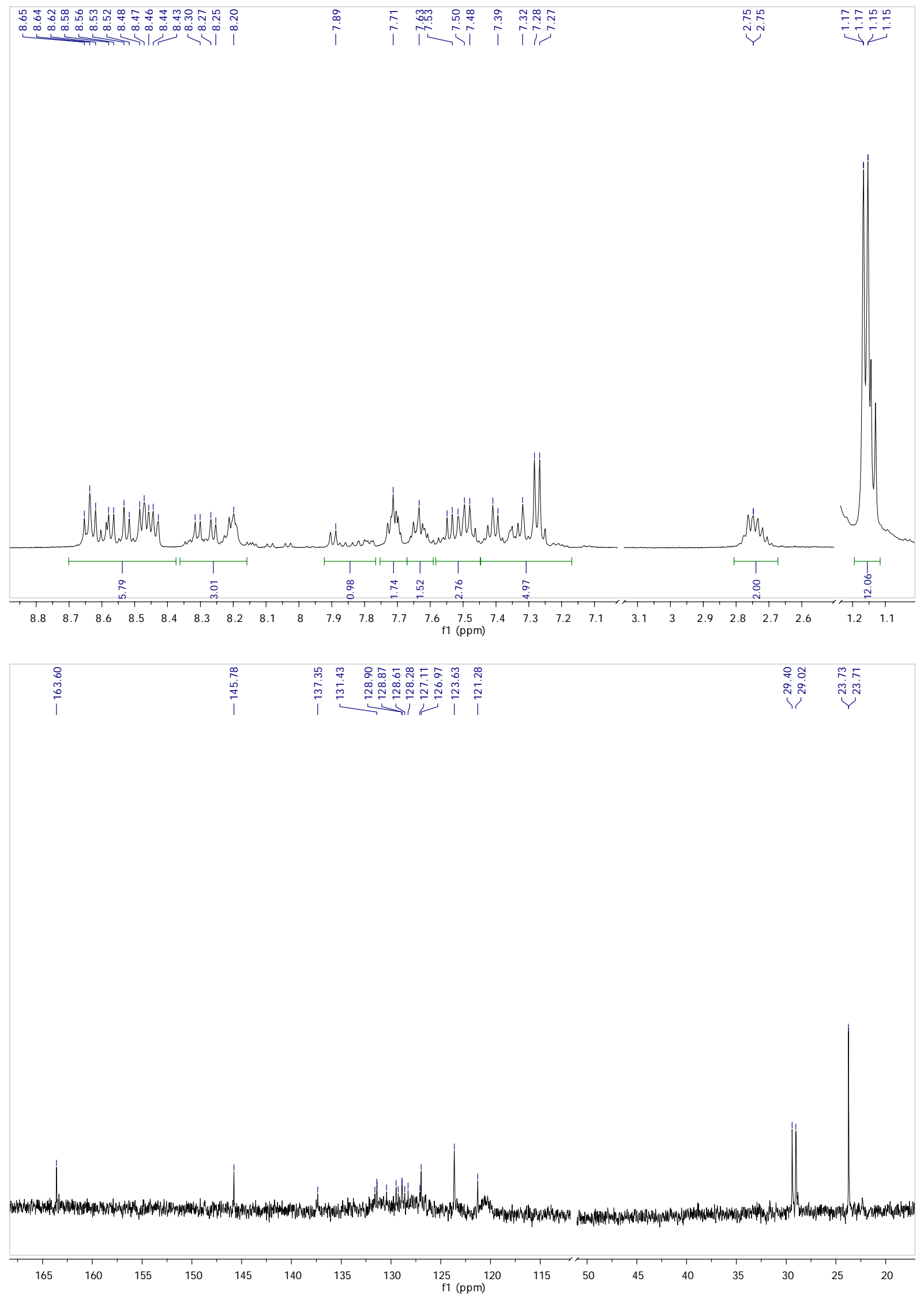

Figure S2. ${ }^{1} \mathrm{H}-\mathrm{NMR}\left(500 \mathrm{MHz}, \mathrm{C}_{2} \mathrm{D}_{2} \mathrm{Cl}_{4}\right)$ and ${ }^{13} \mathrm{C}-\mathrm{NMR}\left(175 \mathrm{MHz}, \mathrm{C}_{2} \mathrm{D}_{2} \mathrm{Cl}_{4}\right)$ spectra of compound $7 \mathbf{a}$. 

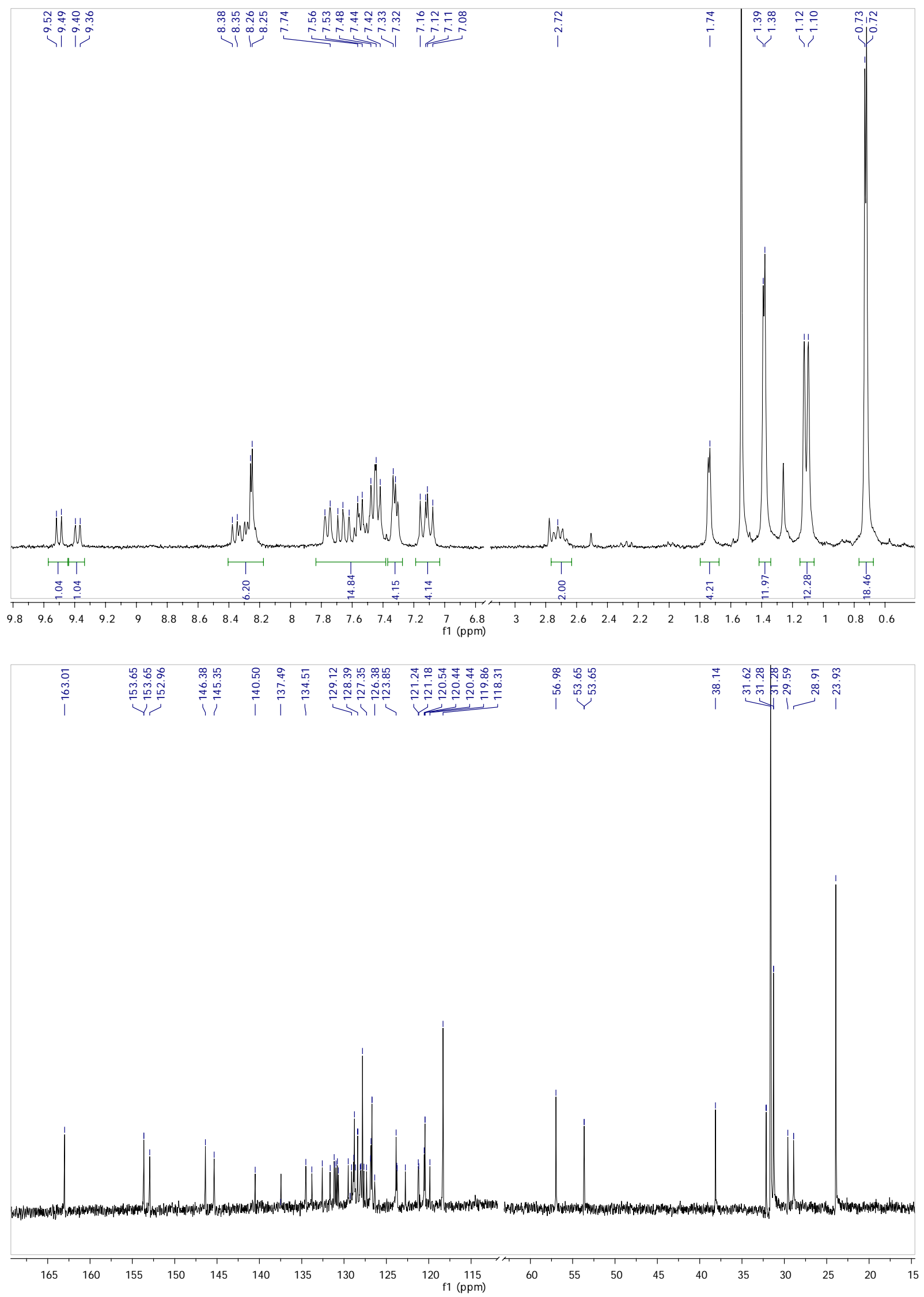

Figure $\mathrm{S} 3 .{ }^{1} \mathrm{H}-\mathrm{NMR}\left(250 \mathrm{MHz}, \mathrm{CD}_{2} \mathrm{Cl}_{2}\right)$ and ${ }^{13} \mathrm{C}-\mathrm{NMR}\left(125 \mathrm{MHz}, \mathrm{CD}_{2} \mathrm{Cl}_{2}\right)$ spectra of compound $7 \mathbf{b}$. 

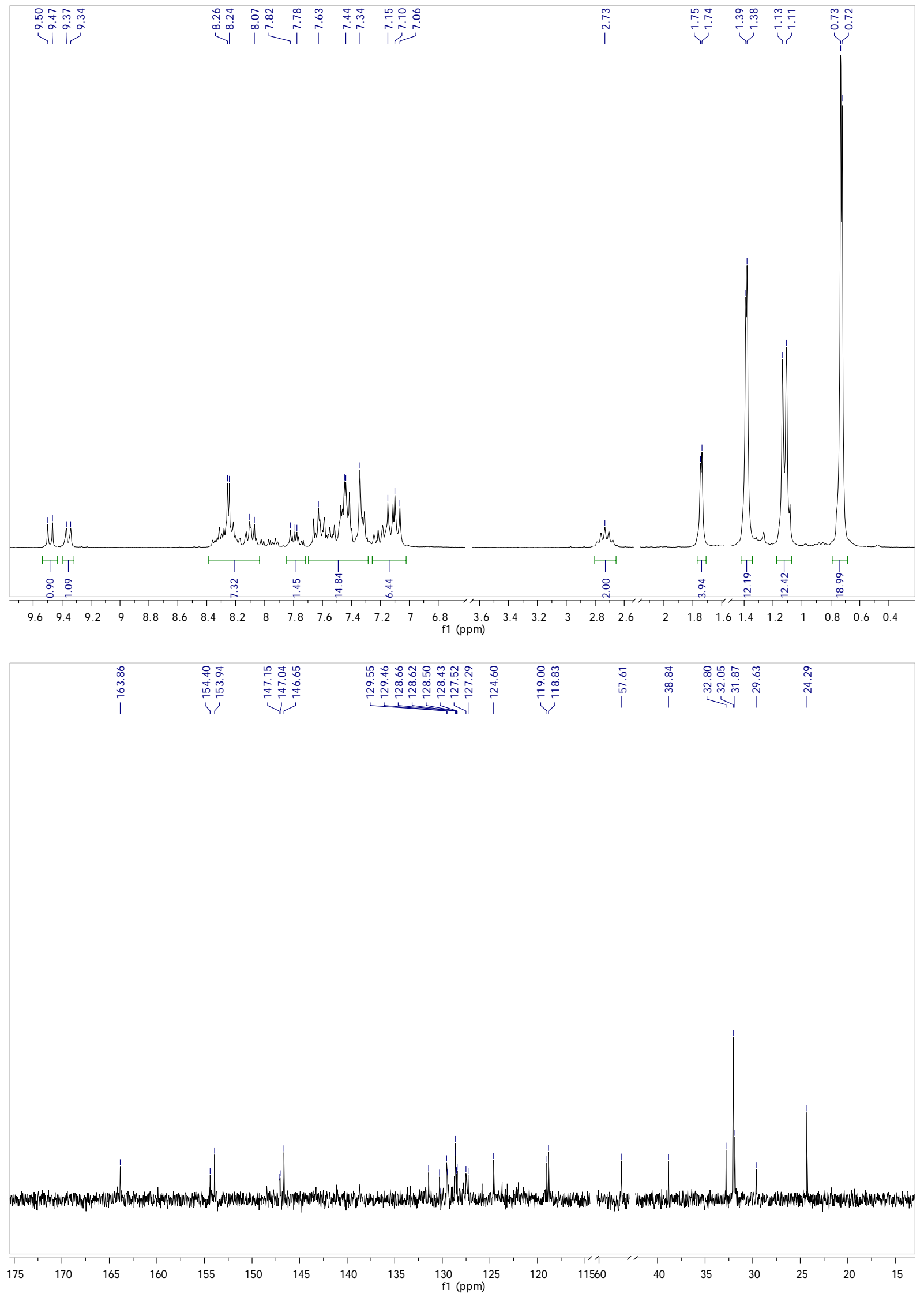

Figure S4. ${ }^{1} \mathrm{H}-\mathrm{NMR}\left(250 \mathrm{MHz}, \mathrm{CD}_{2} \mathrm{Cl}_{2}\right)$ and ${ }^{13} \mathrm{C}-\mathrm{NMR}\left(62.5 \mathrm{MHz}, \mathrm{CD}_{2} \mathrm{Cl}_{2}\right)$ spectra of compound 7c. 

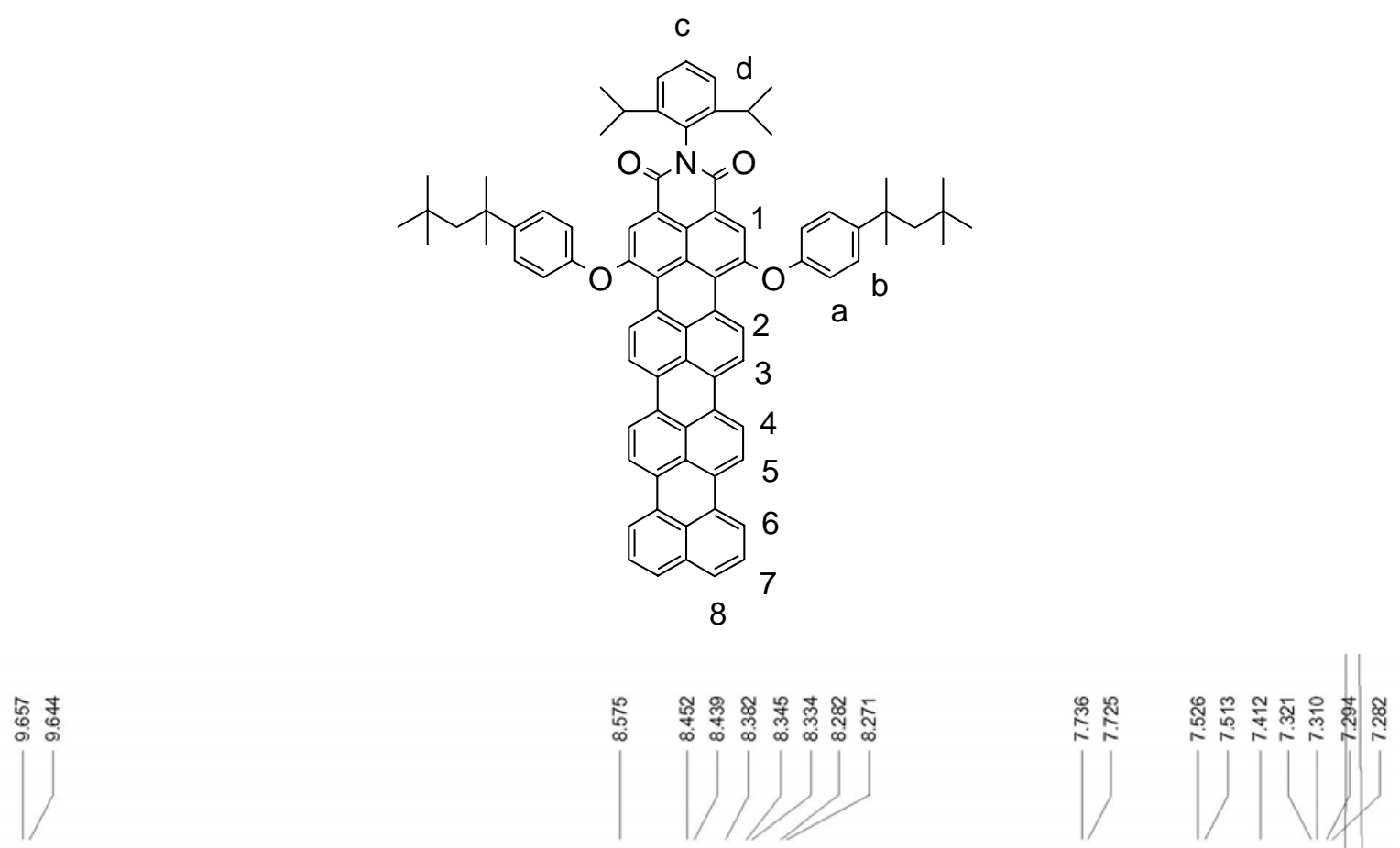

$\mathrm{H}-\mathrm{a}$

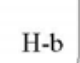

H-4 H-5 H-6

H-2

H-1 H-3

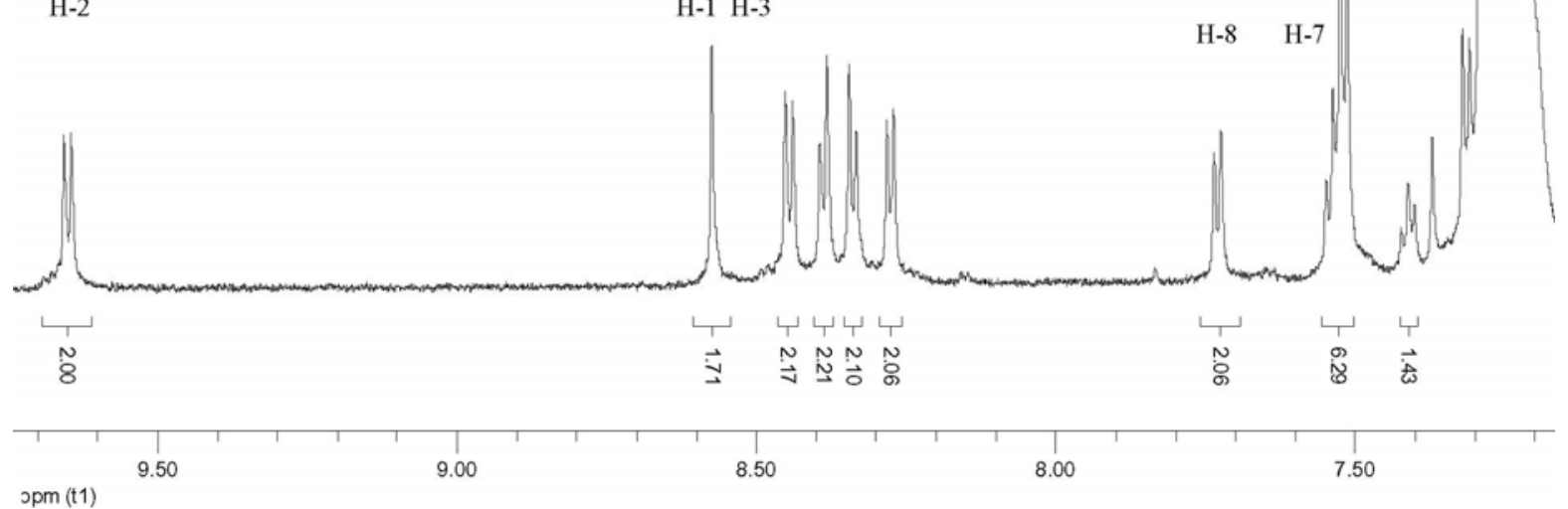

opm (t1)

Figure S5. Aromatic region of ${ }^{1} \mathrm{H}-\mathrm{NMR}$ spectrum of compound $\mathbf{4 b}$ and the assignment of the signals (700 MHz, 1,3,5-trichlorobenzene-d $\mathrm{d}_{3}$ ). 


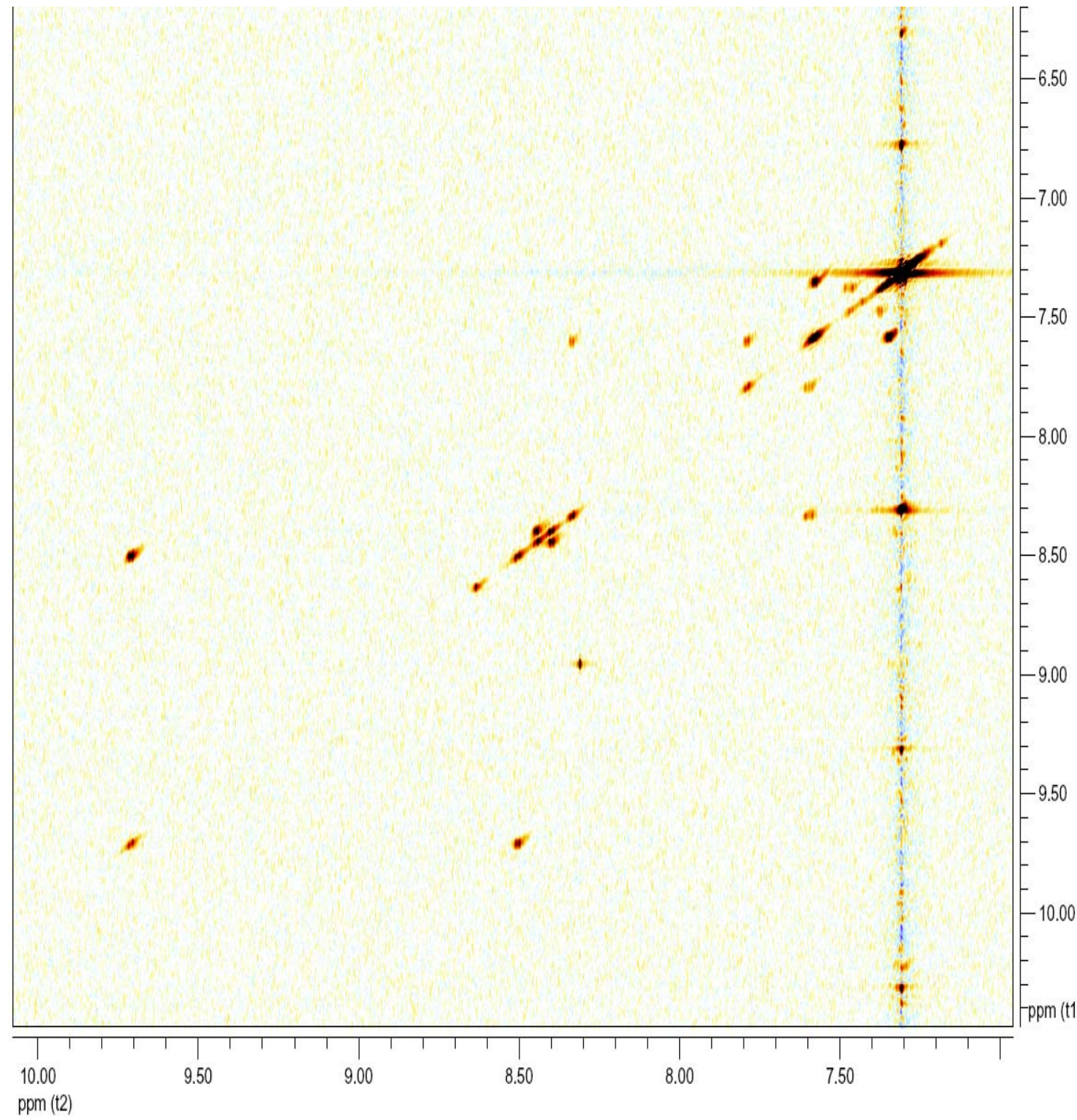

Figure S6. Aromatic region of ${ }^{1} \mathrm{H}-{ }^{1} \mathrm{H}$ COSY-NMR spectrum of compound $\mathbf{4 b}$ (700 MHz, 1,3,5-trichlorobenzene-d $\mathrm{d}_{3}$ ). 

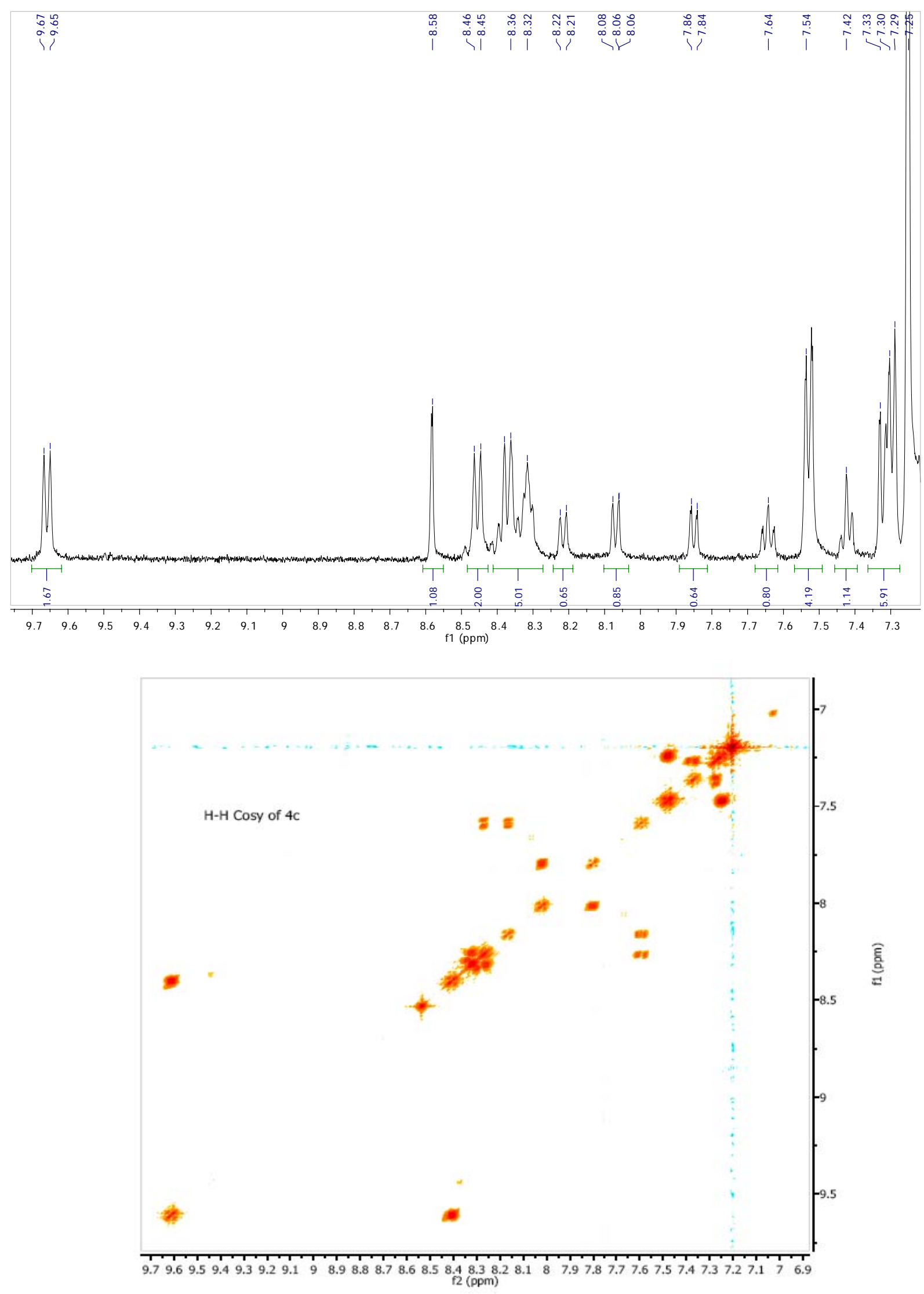

Figure S7. Aromatic region of ${ }^{1} \mathrm{H}-\mathrm{NMR}$ and H-H Cosy-NMR spectra of compound 4c (500 MHz, 1,3,5-trichlorobenzene- $\mathrm{d}_{3}$ ). 